Págs.270-282

\title{
Entrevistas a representantes sindicales
}

\section{Maribel Loranca, secretaria del Sector de Enseñanza del sindicato estatal FeSP-UGT}

Indica, por favor, cuántos miembros estáis conviviendo en casa en este momento de confinamiento, edad de cada miembro, nivel educativo de cada miembro y a qué se dedica, con especial atención a si se está en casa o tiene un empleo que le obliga a salir en este momento (utiliza todo el espacio que consideres oportuno para cada una de las preguntas).

Convivimos dos personas, una de 58 y otra de 69 años. Ambos con formación universitaria. Uno de ellos, ya jubilado y que desinteresadamente presta asistencia telemática en cuestiones jurídicas y laborales. En mi caso, desde el 13 de marzo, estoy en situación de teletrabajo, aunque acudo de manera puntual (una vez por semana) a mi oficina.

Explica brevemente cómo se distribuyen las labores del hogar entre sus miembros durante el confinamiento: tareas que se han realizado en el hogar durante la última semana y duración (aproximada) de cada tarea, así como miembro del hogar que la ha realizado. Señala también si ha cambiado esa distribución de tareas entre los miembros del hogar en relación a antes del confinamiento.

No hemos hecho una distribución explícita. Vamos solventando las tareas día a día. La que más tiempo ocupa es la limpieza porque hemos tomado alguna medida extraordinaria siguiendo las recomendaciones sanitarias. Dedicamos 2 horas diarias para un piso de unos 85 metros cuadrados. Hemos variado la rutina de hacer la compra de la que me encargo yo, ya que soy quien por motivo de trabajo y edad, salgo a la calle. Las demás tareas las organizamos en función de las exigencias de mi trabajo.

Con respecto a la realización del trabajo escolar durante el confinamiento, te pedimos, por favor, que respondas a las siguientes preguntas:

De qué medios tecnológicos dispones en casa (un ordenador, varios ordenadores, una tablet, un móvil inteligente, ninguno de éstos).

Dos ordenadores (uno de ellos cedido para estos días por el sindicato), una tablet y dos smartphone. 
¿Cómo es la conexión a internet que tenéis en tu casa (Tipo de conexión -4G, Fibra, Rural...-, Velocidad)?

Fibra 100 GB.

Desde tu visión como representante sindical, qué se está haciendo desde los centros educativos de Ceuta, por qué vía llegan los deberes, cómo es la interacción entre el profesorado y el alumnado, cómo es la interacción con las familias a la hora de hacer el seguimiento de los deberes, cómo se están teniendo en cuenta las dificultades de las familias con menor nivel educativo o sin acceso a la sociedad digital, cómo se ha planteado la evaluación del $2^{\circ}$ trimestre

Según me comentan mis compañeros de Ceuta, la situación es similar a otras zonas de España. Naturalmente hay zonas de la Ciudad Autónoma de entorno socioeconómico muy desfavorecido (ejemplo, la barriada de El Príncipe) donde las dificultades son importantes. Se ha intentado extender la banda para dotar de conexión wifi a zonas de la ciudad y al parecer, no ha sido posible. El profesorado está procurando mantener el contacto con el alumnado por todas las vías posibles, predominando el contacto por teléfono cuando no es posible por otro medio.

Con respecto a la evaluación, se está a la espera de las instrucciones del Ministerio de Educación y Formación Profesional.

\section{Indica si crees que esta forma de seguir el curso está teniendo algún impacto en el aprendizaje del alumnado y en qué sentido}

Indudablemente, sí. La situación de emergencia sanitaria ha puesto en evidencia de manera cruda, la brecha educativa en nuestro país, que desde luego es producto de la brecha social. Aún así, yo distinguiría distintos aspectos: en determinadas etapas y cursos, solamente debería preocuparnos que los niños y los adolescentes no pierdan el vínculo con el centro educativo y en el caso de los segundos, la «tensión» del proceso, el hábito de estudio y no se relajen.

En los cursos que conducen a titulación, la incidencia no es tanto porque adquieran o no, determinados conocimientos, sino por la incertidumbre que están viviendo y el estrés al que están sometidos.

Esta situación ha agravado además la pérdida de equidad que nuestro sistema educativo viene sufriendo en los últimos años por los recortes sufridos.

\section{Señala las dificultades que os están llegando, como sindicato, por parte de profesora-} do, familias y otros posibles agentes educativos.

Depende mucho de las circunstancias. No voy a detenerme en quienes se mueven en un buen contexto socioeconómico y cultural buenos.

En general, profesorado, familias y alumnado están sometidos a un estrés brutal. El profesorado ha tenido que reinventar en tiempo récord otra forma de impartir clase y de relacionarse con los alumnos. Lo ha hecho en general, aportando sus medios personales y sin orientaciones claras y sin pautas de desconexión digital, sintiéndose continuamente escrutados y evaluados.

En cuanto a las familias, muchas han perdido sus posibilidades de conciliar vida laboral y familiar. Para quienes tienen la suerte de teletrabajar, está siendo muy complicado poderlo hacer con los niños en casa. Quienes se ven forzados a salir para realizar su trabajo tampoco tienen redes de apoyo y por último, quienes han perdido su trabajo viven una doble situación de estrés por la incertidumbre de su futuro. 
Cuáles son las principales conclusiones que extraes de esta experiencia educativa desde tu posición como sindicalista.

Más que nunca, nuestro país debe reflexionar sobre qué modelo de sociedad tenemos y cuál queremos tener. Los últimos 8 o 10 años han supuesto para nuestro Estado de Bienestar (con sus tres pilares: Educación, Sanidad y Servicios Sociales) un retroceso que será muy difícil recuperar. La situación provocada por la emergencia sanitaria que es ahora la absoluta prioridad, puede agravar la situación de deterioro que hemos vivido en los últimos años. Urge un acuerdo nacional para evitar el aumento de la brecha social y poder ir recuperando niveles de equidad.

Señala si crees que está teniendo algún impacto en términos de igualdad o desigualdad para el alumnado y, en caso afirmativo, en qué sentido.

Por supuesto, la brecha digital y educativa que ya existía se ha manifestado con crudeza. La actividad presencial permitía intentar paliar las desigualdades. Cuando ésta se paraliza y el proceso solamente puede hacerse a distancia, el desigual acceso del alumnado a las TIC puede hacer retroceder a un número importante de alumnos e incluso, hacerles abandonar el sistema educativo.

Ahora te pedimos que hagas un breve balance de esta experiencia de confinamiento y de teletrabajo educativo con relación a las siguientes cuestiones:

Principales debilidades y dificultades, situaciones de estrés, de conflicto...

Me referiré al teletrabajo sindical. Los principales problemas en mi caso son la imposibilidad de la desconexión digital, las dificultades para el trabajo en equipo y la impotencia por no estar físicamente al lado de los trabajadores que lo están pasando mal. Que no puedan sentir el aliento y el apoyo de los responsables sindicales.

Aspectos positivos, algo que puedas extraer como una ventaja en medio de esta situación compleja, alguna reflexión en términos de crecimiento para la sociedad y para el sistema educativo.

La capacidad de adaptación y de reinventarnos como profesionales. En el ámbito educativo, el convencimiento de que se van a sacar muchas conclusiones de esta situación y se van a repensar cómo debe ser el perfil de los futuros docentes y los excesivos currículos. Socialmente, debería llevarnos a trabajar contra la brecha social. Si no, seguirá existiendo la brecha digital y por supuesto, la educativa.

\section{Y en términos emocionales, afectivos y personales, ¿̇cómo lo estás viviendo? ¿̇cómo lo estás gestionando?}

Tengo la suerte de pasar el confinamiento en compañía y tener medios tecnológicos para mantener contacto con familia y amigos. Procuro combatir el estrés (que algunos días es mucho) con algo de actividad física (bicicleta estática), lectura y cine. Aun partiendo de una situación cómoda, hay días que no resulta sencillo. Reconozco que también me ayuda el día que salgo para ir a mi centro de trabajo.

\section{Qué aprendizaje extraes de esta experiencia de cara al futuro.}

Aunque suene a tópico, creo que esto nos recuerda la distinción entre cosas urgentes e importantes y que desde luego, podemos vivir felizmente con muchas menos cosas que, en circunstancias normales, consideramos necesarias. 
Te pedimos también que valores las consecuencias y el impacto educativo y social de esta crisis a corto y medio plazo, una vez que se supere la situación.

Es una cuestión que me causa bastante preocupación. Creo que aún no somos conscientes de la envergadura de la crisis que se nos puede venir encima. Sería un error que una vez normalizada la situación, pretendamos seguir como si nada hubiera pasado. La recuperación implicará un esfuerzo colectivo que no puede recaer solamente en aquellos que ya sufrieron los peores efectos de la crisis económica que arrancó en 2008. Estábamos recuperándonos lentamente, pero se quedó demasiada gente atrás.

En el ámbito educativo, maltrecho por los recortes sufridos, es necesario más que nunca, un esfuerzo presupuestario que no sé si el país va a estar en condiciones de hacer.

Finalmente, nos gustaría preguntarte por alguna otra cuestión, comentario, sugerencia, que quieras hacer para terminar desde tu posición como responsable sindical a nivel estatal, lo que te confiere una visión especialmente relevante en este momento tan complicado para la sociedad y para la educación en España.

Sugerencias: Pacto Estatal por la recuperación que debe abordar distintos aspectos. Fiscalidad progresiva, justa y redistributiva y blindar los pilares del Estado de Bienestar.

Compromiso con la recuperación del país por parte del sistema financiero, la gran empresa, la clase política y los agentes sociales. 


\section{Representante sindical, 58 años, Universidad de Granada}

Indica, por favor, cuántos miembros estáis conviviendo en casa en este momento de confinamiento, edad de cada miembro, nivel educativo de cada miembro y a qué se dedica, con especial atención a si se está en casa o tiene un empleo que le obliga a salir en este momento (utiliza todo el espacio que consideres oportuno para cada una de las preguntas).

Yo vivo solo.

Explica brevemente cómo se distribuyen las labores del hogar entre sus miembros durante el confinamiento: tareas que se han realizado en el hogar durante la última semana y duración (aproximada) de cada tarea, así como miembro del hogar que la ha realizado. Señala también si ha cambiado esa distribución de tareas entre los miembros del hogar en relación a antes del confinamiento.

Lógicamente yo hago todas las labores de la casa.

Con respecto a la realización del trabajo durante el confinamiento, te pedimos, por favor, que respondas a las siguientes preguntas:

De qué medios tecnológicos dispones en casa (un ordenador, varios ordenadores, una tablet, un móvil inteligente, ninguno de éstos).

Un ordenador portátil, una tablet y dos móviles.

¿Cómo es la conexión a internet que tienes en tu casa (Tipo de conexión-4G, Fibra, Rural...-, Velocidad) $\dot{2}$

Mi conexión es de fibra a $100 \mathrm{Mb}$.

Desde tu visión como representante sindical, cómo valoras la respuesta que se está dando desde la universidad.

Creo que la respuesta por parte de la Universidad es increíble, pues se ha conseguido de un día a otro, poder mantener las actividades que se estaban realizando.

Por el contrario, yo echo en falta que se haya permitido la colaboración de los representantes de los trabajadores, lo cual hubiera permitido una mejor compenetración y una mejor utilización de los recursos.

Creo que en general, estamos acercándonos a algo similar al depotismo ilustrado, es decir, no preguntar las necesidades y capacidades de las personas que han tenido que sacar adelante la situación y, por contra, poner en duda en todo momento que los trabajadores de la Universidad quieren hacer las cosas lo mejor posible.

Indica si crees que esta forma de seguir el curso está teniendo algún impacto en el aprendizaje del alumnado y en qué sentido.

Por supuesto que está teniendo impacto, y mucho, hay que tener en cuenta que no todo el alumnado está en las mismas situaciones, y en muchos casos, este sistema va a provocar situaciones discriminatorias, fundamentalmente por criterios económicos, pero también por criterios geográficos o sociales. 
De todas formas, el mayor problema aparecerá al final del curso con la evaluación de los conocimientos adquiridos, pues, como todo el mundo dice, no hay forma segura de evaluar a un conjunto elevado de estudiantes, en materias donde es necesario evaluar el dominio en problemas o casos prácticos de forma igualitaria.

Señala las dificultades que os están llegando, como sindicato, por parte de profesorado, estudiantes y otros posibles agentes educativos.

Fundamentalmente, por parte del profesorado las mayores quejas están centradas en el gran incremento del trabajo a realizar, donde en muchos casos esta actividad está llenando casi por completo no solo la jornada laboral, sino también, lo que correspondería al tiempo libre.

Esto, en muchos casos, está a llevando a situaciones de estrés en mayor o menor medida.

Otro aspecto importantísimo, está centrado en los problemas de conciliación familiar, ya que al tener a los niños en casa es complicado mantener la actividad académica.

Por parte del alumnado, aunque no nos llegan directamente sus quejas, parece que están más centradas en la falta de medios adecuados para seguir la docencia con los nuevos sistemas, (falta de ordenadores, necesidades de conexiones de red, etc.).

Cuáles son las principales conclusiones que extraes de esta experiencia educativa desde tu posición como profesor y como representante sindical. Aquí nos interesa especialmente conocer tu opinión sobre el impacto de la pandemia y la virtualización de una universidad presencial sobre el profesorado a corto y medio plazo.

No creo que todavía seamos capaces de evaluar todas las consecuencias que se van a derivar de la pandemia. Es bueno lo que se ha podido mejorar en la virtualización de la docencia, pero debido a que todo se ha realizado a la carrera, hay cosas que no se han podido hacer bien, y que en muchos casos, va a llevar a un regreso a la actividad anterior.

Señala si crees que está teniendo algún impacto en términos de igualdad o desigualdad para el alumnado y, en caso afirmativo, en qué sentido.

Como he dicho antes, es ineludible que se van a producir desigualdades grandes, fundamentalmente debidas a las situaciones económicas y sociales. No podemos olvidar, que la pandemia ha colocado a muchas familias en situaciones enormemente complicadas, y que lógicamente no van a poner en primer lugar la educación (medios necesarios), cuando la prioridad es poder comer cada día.

Ahora te pedimos que hagas un breve balance de esta experiencia de confinamiento y de teletrabajo educativo con relación a las siguientes cuestiones: Principales debilidades y dificultades, situaciones de estrés, de conflicto...

Podríamos citar como ya he citado anteriormente las situaciones de conciliación familiar, aunque no podemos olvidar que todo el mundo necesita la capacidad de moverse y relacionarse y, de diferentes formas estoy seguro de que todos sentimos un cierto estrés por el prolongamiento del confinamiento. 
Aspectos positivos, algo que puedas extraer como una ventaja en medio de esta situación compleja, alguna reflexión en términos de crecimiento para la sociedad y para el sistema educativo.

Creo que como aspecto positivo hay que citar el nivel de colaboración entre las personas, creo que casi todos dudábamos de que la población española fuera capaz de soportar un largo confinamiento como lo ha estado realizando.

\section{Y en términos emocionales, afectivos y personales, ¿̇cómo lo estás viviendo? ¿̇cómo lo estás gestionando?}

Quizás, al vivir anteriormente solo, me ha resultado un poco más sencillo, pero de todas maneras, ya empiezo a necesitar, como supongo que todos, el regreso a la actividad normal. Lógicamente, he aumentado el número de contactos vía telefónica y de internet con familiares y amigos, pero siempre hechas en falta algo más.

\section{Qué aprendizaje extraes de esta experiencia de cara al futuro.}

Fundamentalmente, que no hay que dar por supuesto nada, porque hemos podido ver que una actividad perfectamente planificada, se puede ir al traste y tener que improvisar soluciones inmediatas.

Te pedimos también que valores las consecuencias y el impacto educativo y social de esta crisis a corto y medio plazo, una vez que se supere la situación.

Personalmente, creo que estamos ante un curso totalmente atípico. Dado que todo el mundo cuando hace referencia a la evaluación cita conceptos como flexibilidad, da una idea de que todo el mundo piensa que el nivel de conocimientos adquiridos no va a ser el adecuado. Yo me pregunto si en un país donde la capacidad de ejercer diferentes profesiones la otorga la Universidad, si nos podemos permitir profesionales que no hayan adquirido los conocimientos necesarios.

Respecto al impacto social, no estoy todavía en condiciones de saberlo, pero tengo la percepción de que el efecto va a ser muy importante, llevándonos a grandes modificaciones de nuestro estilo de vida. 


\section{Ferran Vicent Garcia Ferrer, 53 años, áreas de Cultura y Educación de CCOO País Va- lencià y Profesor Asociado, Universidad de Valencia}

Las opiniones escritas aquí son fruto de la valoración y experiencia personal y no representan necesariamente la opinión del sindicato.

Indica, por favor, cuántos miembros estáis conviviendo en casa en este momento de confinamiento, edad de cada miembro, nivel educativo de cada miembro y a qué se dedica, con especial atención a si se está en casa o tiene un empleo que le obliga a salir en este momento (utiliza todo el espacio que consideres oportuno para cada una de las preguntas).

Convivimos cuatro personas.

Mi hijo de 23 años. Estudiante de Máster post Grado en Física. Está en casa.

Mi hija de 25 años. Grado en Filosofía. Sindicalista. Salidas muy ocasionales por atención al trabajo.

Mi compañera de 53 años. Estudios técnicos medios en cerámica y diseño gráfico. Trabaja en Centros de Atención a Mayores y producción cerámica. Salidas ocasionales a su taller de cerámica y pintura para trabajar.

Y yo, de 53 años. Doctor en Física. Sindicalista. Profesor asociado en la Facultad de Física, con 3 horas semanales. Salidas muy ocasionales por atención al trabajo de sindicalista.

Explica brevemente cómo se distribuyen las labores del hogar entre sus miembros durante el confinamiento: tareas que se han realizado en el hogar durante la última semana y duración (aproximada) de cada tarea, así como miembro del hogar que la ha realizado. Señala también si ha cambiado esa distribución de tareas entre los miembros del hogar en relación a antes del confinamiento.

Última semana:

Hijo: lavadoras, 5 horas semanales.

Hija: limpieza de lavabos y cocina, 5 horas semanales.

Mi compañera: limpieza de polvo y general de la casa, 5 horas semanales.

Yo: lavavajillas, 5 horas semanales.

Estas tareas son rotativas cada semana, antes del confinamiento y también durante el confinamiento.

Compra semanal: dos viajes. Uno mi compañera y otro yo. Entre 2 y 3 horas. Fuera de confinamiento solo iba ella a comprar.

Comidas: mi compañera. 1,30 horas diarias.

Cenas: yo. 1 hora diaria.

Antes del confinamiento, ella hacía las dos comidas diarias.

Con respecto a la realización del trabajo durante el confinamiento, te pedimos, por favor, que respondas a las siguientes preguntas:

De qué medios tecnológicos dispones en casa (un ordenador, varios ordenadores, una tablet, un móvil inteligente, ninguno de éstos).

Para mi uso personal: un ordenador de mesa, un ordenador portátil y un smartphone.

El resto de personas de la casa, cada una tiene un ordenador portátil, una tablet y un smartphone. 


\section{¿Cómo es la conexión a internet que tienes en tu casa (Tipo de conexión -4G, Fibra, Rural...-, Velocidad)?}

Conexión wifi por fibra de 100Mb.

\section{Desde tu visión como representante sindical, cómo valoras la respuesta que se está dando desde la universidad.}

La universidad, al igual que la mayoría de centros de trabajo de titularidad pública, ha mostrado tanto la buena voluntad de muchas de las personas que la coordinan y dirigen, como una velocidad de respuesta a nuevas situaciones claramente lenta.

Pienso que la situación actual exigía dos nuevos planteamientos principalmente:

El primero, mantener y aumentar la inversión pública y el esfuerzo contractual para minimizar el impacto en la economía productiva y en las rentas familiares. En este aspecto, a las mentalidades con menor visión social de la economía les ha costado asumir que el objetivo era superior a la relación capital-trabajo y que era necesario mantener sueldos y contratos a pesar de la disminución o parada de producción. Ha sido más un problema de velocidad de respuesta en cada ámbito que la dinámica global. En niveles globales, la universidad pública ha sido responsable y ha mantenido estas relaciones contractuales.

El segundo, mantener a las personas en sus casas al máximo nivel posible. Creo que la universidad ha actuado de acuerdo con el pensamiento social mayoritario. Ni ha ido por detrás de la percepción social mayoritaria -al principio no se le dio mayor importancia al confinamiento- y tampoco por delante -como se podría suponer por bagaje cultural y científico-.

Al principio, las recomendaciones fueron laxas, para, finalmente, y de forma generalizada, suspender las clases presenciales y la asistencia de todo personal a las instalaciones universitarias de acuerdo con las directrices en materia sanitaria del Gobierno.

\section{Indica si crees que esta forma de seguir el curso está teniendo algún impacto en el aprendizaje del alumnado y en qué sentido.}

El aprendizaje basado en la asimilación de contenidos (conceptuales y procedimentales) a partir de la impartición de clases presenciales y de lectura dirigida de textos se ha visto muy limitado en su avance por esta situación.

El aprendizaje de «aprender a aprender» en cada ámbito de las enseñanzas superiores se puede ver reforzado por la novedad de los recursos y necesidades que se han planteado.

El confinamiento ha limitado nuestro acceso a la interacción personal -preguntas y respuestas presenciales y en grupo- y también a los recursos bibliográficos.

Con todo ello, se podría avanzar en cambiar la visión pedagógica del aprendizaje para adquirir mayores competencias en aprender en este contexto y con estos recursos escasos y novedosos.

En este sentido, solamente el alumnado y profesorado que miren la forma de cambiar esos objetivos mejoraran respecto de la situación anterior. Quienes se mantengan en el paradigma de clase magistral y lectura de textos, simplemente perderán parte de las enseñanzas curriculares. 
Señala las dificultades que os están llegando, como sindicato, por parte de profesorado, estudiantes y otros posibles agentes educativos.

Ha habido gran sensación de indefensión frente a la vulnerabilidad ante un contagio por exceso de exigencia laboral y también de escasez de recursos para atender las demandas que la sociedad nos impone (al profesorado, a los alumnos y a los agentes educativos) desde la distancia y el confinamiento.

Puedo destacar:

- Lentitud de respuesta organizada pedagógicamente por parte de los equipos docentes.

- Lentitud de la exigencia de no asistir al centro de trabajo por parte de los equipos directivos.

- Lentitud en la constatación y adaptación a los medios disponibles para el teletrabajo.

- Escasa cultura de adaptación y modificación de los objetivos a conseguir y los medios utilizados tanto en el ámbito de la investigación como en el de la docencia.

- Presencia abundante en el ámbito de las decisiones personales y opiniones comunes de la mentalidad liberal laboral por la cual en situación de menor o más difícil prestación del servicio había que reducir o eliminar las relaciones contractuales y salariales.

Cuáles son las principales conclusiones que extraes de esta experiencia educativa desde tu posición como profesor y como representante sindical. Aquí nos interesa especialmente conocer tu opinión sobre el impacto de la pandemia y la virtualización de una universidad presencial sobre el profesorado a corto y medio plazo.

La relación presencial ofrece una mejor atención, más eficaz y económica -en el amplio sentido de la palabra- que la relación virtual y a distancia.

Por tanto, si no hay fuerza mayor que la impida, deberíamos tender a recuperarla.

En cualquier caso, esta virtualización «a la fuerza» nos debería enseñar a adaptar objetivos pedagógicos y productivos a una mayor capacidad de decisión, organización y planificación por parte de todas las personas y niveles implicados. Empezando por las dinámicas de planificación personal asociadas a la capacidad de proponerse objetivos propios y extendiéndose a los ámbitos organizativos desde los más cercanos y primarios hasta los más elevados y coordinados.

La mejora en la capacidad de las personas y organizaciones de relacionarse a través de medios de comunicación en red puede ser también un efecto de esta situación. Todavía es fácil encontrar en ciertos ámbitos desconfianza o reticencias para integrarse y utilizar con asiduidad la tecnología asociada a este tipo de comunicación. La situación de recurrir por fuerza a los medios TIC puede haber rebajado o hecho desaparecer muchas de estas barreras.

Queda por resolver una valoración y planificación global de cuál es el acceso a esos medios y la mejora necesaria tanto desde el punto de vista social como universitario.

\section{Señala si crees que está teniendo algún impacto en términos de igualdad o desigual- dad para el alumnado y, en caso afirmativo, en qué sentido.}

El alumnado puede ver aumentada la desigualdad en función de dos factores:

- El acceso a los medios TIC. Tanto en el conocimiento y destrezas necesarios, como en los recursos disponibles. Menor acceso implica mayor tiempo de dedicación para obtener menores resultados.

- El acceso a la atención educativa. No todas las personas tienen la misma posibilidad de ser acompañadas en el proceso de enseñanza y aprendizaje. Sus conexiones sociales son claves en este aspecto. 
Algunas personas pueden ser asesoradas por una red efectiva de otras personas capacitadas educativa y culturalmente y otras, en cambio, ven muy limitado su acceso a ese asesoramiento o llegan a carecer de él, totalmente. La reducción de la atención educativa por escasa interacción social será otro factor de desigualdad que aumentará, ya que no es paliado por la asistencia a lugares de reunión comunes como son los centros educativos.

Ahora te pedimos que hagas un breve balance de esta experiencia de confinamiento y de teletrabajo educativo con relación a las siguientes cuestiones: Principales debilidades y dificultades, situaciones de estrés, de conflicto...

Fisiológicamente tengo muchos problemas con la espalda. Para evitarlos, o reducirlos, practico deporte seis veces por semana, en sesiones de una hora u hora y media. Deportes al aire libre como correr, bicicleta y natación.

Esta limitación es la más notable físicamente. El ejercicio en casa (sin acceso a ningún espacio abierto y en un apartamento de $90 \mathrm{~m}^{2}$ en un segundo piso) puede paliar la pérdida de forma física y los dolores, pero sigue siendo la principal dificultad que presenta para mí esta situación.

Otro conflicto proviene de las dificultades para establecer tiempos de descanso y trabajo. Al trabajar con herramientas de comunicación en red la sensación es de estar disponible todas las horas, todos los días. Muchas personas han asumido que es así, que no hay diferenciación en los tiempos, y, por tanto, la interacción con mis grupos de trabajo también me lleva a más dificultades en ese ámbito. Considero imprescindible mantener esos ritmos de cambio de actividad diaria, semanal y mensualmente. Sin una distribución correcta de trabajo, ejercicio y descanso no creo que se puedan mantener los niveles de atención que mi trabajo (sindical y educativo) requiere.

Hay también una interacción social rutinaria que facilita la relajación del estrés mental y favorece la creatividad y propuesta de líneas de trabajo o de tiempo libre. Esta ahora está muy limitada a las «quedadas» virtuales, muy reducidas en frecuencia, en número de personas y en duración. Cabe añadir que la principal comunicación pasa a ser escrita en diversos chats y eso añade barreras y esfuerzo a la relación interpersonal.

Por último, la atención médica habitual también se ha visto interrumpida y la visita al médico de cabecera, fisioterapeuta, traumatólogo, dentista, etc. para consultas de mantenimiento no es posible. Aumenta, por tanto, la sensación que cuando acabe el confinamiento, habrá que dedicar tiempo y esfuerzo a recuperarse de los deterioros sufridos.

\section{Aspectos positivos, algo que puedas extraer como una ventaja en medio de esta situa- ción compleja, alguna reflexión en términos de crecimiento para la sociedad y para el sistema educativo.}

Una enseñanza para nuestra relación con el medio ambiente y nuestro entorno: sería mejor buscar la forma de cambiar nuestros hábitos fuertemente consumistas de invadir en tropel las zonas con mayor riqueza paisajística, de utilizar en exceso los vehículos a motor privados, los viajes de larga distancia y corta duración, etc. para establecer una menor presión sobre el entorno, la vida del ecosistema y el calentamiento global.

La economía y las dinámicas sociales deben ser reorientadas hacia el consumo de proximidad y formas de descanso y disfrute del tiempo libre menos masificadas. 
Otra posible aportación, ésta en el ámbito educativo, sería el focalizar más en la competencia de «aprender a aprender» en contextos diferentes y exigentes y menos en la adquisición de contenidos. En el mismo ámbito, priorizar la capacidad de auto-organización y planteamiento de propuestas para mejorar la agilidad en la respuesta a diversas situaciones y retos sociales.

En el ámbito económico y político, se debería valorar que las mejores respuestas sanitarias y sociales solo han sido posibles con una organización estatal fuerte y servicios sociales públicos también sólidos y amplios. La iniciativa privada no tiene capacidad para coordinar una respuesta tan exigente y total como la necesaria en una crisis de esta magnitud. No parece imposible que en un futuro próximo nos enfrentemos a otras de este alcance y, por tanto, deberíamos actuar para reforzar el ámbito de los recursos y la gestión públicas frente al poder de las corporaciones privadas.

Este cambio de tendencia y de mentalidad, nos debe guiar en los ámbitos nacionales, autonómicos, municipales y también europeo e incluso planetario. La esfera de lo público no puede perder la batalla de la globalización frente a la iniciativa privada lucrativa si queremos tener defensa frente a amenazas globales como la actual.

Y, volviendo al terreno más personal, la gestión de los tiempos de descanso, trabajo, atención médica y relación social debe ser una de las habilidades y dedicaciones preferentes para la salud de la población en general.

\section{Y en términos emocionales, afectivos y personales, ¿̇cómo lo estás viviendo? ¿cómo lo estás gestionando?}

Creo que he respondido esta pregunta de forma suficientemente amplia en las preguntas anteriores en lo relativo a los siguientes ámbitos afectados por el confinamiento:

- Práctica laboral sindical.

- Práctica educativa.

- Gestión del ejercicio físico y la salud.

- Relaciones sociales y disfrute del tiempo libre.

\section{Qué aprendizaje extraes de esta experiencia de cara al futuro.}

Esta pregunta ya la he respondido previamente, dado que planteo las mismas enseñanzas para mí que para la sociedad en la que vivo.

Te pedimos también que valores las consecuencias y el impacto educativo y social de esta crisis a corto y medio plazo, una vez que se supere la situación.

El impacto a corto y medio plazo dependerá en gran parte de nuestra capacidad de incorporar aprendizajes de forma crítica y constructiva a partir de cómo hemos reaccionado ante una situación de emergencia excepcional.

Si somos capaces de avanzar en mejorar nuestra capacidad de auto-organizarnos desde los niveles más sencillos a los más complejos, si priorizamos la capacidad de aprender a aprender, si contamos con una relación educativa constructiva donde la relación entre quien enseña y quien aprende se hace más simétrica, se consolidaran las mejoras que hemos incorporado con nuestro esfuerzo en estos días. Podemos ser más interactivos, superar mejor las distancias interpersonales, utilizar mejor nuestra capacidad de propuesta didáctica y estar más atentos a las desigualdades de acceso a la educación del alumnado, todo depende de nosotros y nosotras. 
Respecto al impacto social, creo que también debemos reflexionar sobre el carácter claramente privado de las comunicaciones en red. Tenemos medios de comunicación públicos para el transporte, para las comunicaciones por televisión y radio, etc. pero las redes de transmisión de datos y las aplicaciones tecnológicas para socializar nuestra comunicación pertenecen a empresas privadas. Las diferentes crisis que han surgido con la protección de datos y también con la veracidad de contenidos nos deben llevar a una reflexión sobre la necesidad de tener redes, canales y recursos tecnológicos públicos.

Finalmente, nos gustaría preguntarte por alguna otra cuestión, comentario, sugerencia, que quieras hacer para terminar desde tu posición como responsable sindical en la universidad, lo que te confiere una visión especialmente relevante en este momento tan complicado para la sociedad y para la educación en España.

Si debo destacar alguna reflexión desde ese punto de vista, me quedo con dos apuntes, uno desde las relaciones laborales y otro desde el modelo pedagógico de educación superior:

En las relaciones laborales, las universidades públicas deberían asumir su papel de liderazgo. Deben mantener derechos laborales en todas las formas de relación y contratación existentes, así como el respeto a la salud y prevención de riesgos que sirvan de modelo a otras instituciones del ámbito educativo y de la investigación.

En el ámbito educativo, se debe avanzar hacia un modelo que potencie la capacitación profesional de las personas que se forman en los centros universitarios. Una mejor capacitación profesional en todas las enseñanzas del espacio superior europeo pasa por conseguir que el alumnado incorpore competencias para la capacidad de elaborar propuestas para el desarrollo y la innovación, mas allá de la capacitación técnica correspondiente y necesaria. 